\title{
Adenoviral-mediated Transfer of the Human Endothelial Nitric Oxide Synthase Gene Reduces Acute Hypoxic Pulmonary Vasoconstriction in Rats
}

\author{
Stefan P. Janssens, ${ }^{\star}$ Kenneth D. Bloch, ${ }^{\ddagger}$ Zengxuan Nong, ${ }^{\star}$ Robert D. Gerard, ${ }^{\star}$ Pierre Zoldhelyi, ${ }^{\star}$ and Desire Collen* \\ *Cardiac Unit and the Center for Transgene Technology and Gene Therapy, University of Leuven and Flanders Interuniversity Institute \\ for Biotechnology, Belgium; and ${ }^{\ddagger}$ Cardiovascular Research Center, Massachusetts General Hospital, Harvard Medical School, \\ Boston, Massachusetts 02115
}

\begin{abstract}
Nitric oxide (NO), a vasodilator involved in the regulation of pulmonary vascular tone, is synthesized by a family of enzymes, nitric oxide synthases (NOS). To investigate whether adenoviral-mediated overexpression of constitutive endothelial NOS (ceNOS) would attenuate hypoxic pulmonary vasoconstriction, we aerosolized $3 \times 10^{9}$ plaque forming units of a recombinant adenovirus containing the ceNOS gene (AdCMVceNOS) into rat lungs. Four days after infection, transgene expression was confirmed using immunoblot techniques. Diffuse ceNOS immunostaining was detected in alveoli and medium-sized and small pulmonary vessels of AdCMVceNOS-transduced lungs. AdCMVceNOStransduction was associated with an $86 \%$ increase in $\left[{ }^{3} \mathrm{H}\right]$ arginine to $\left[{ }^{3} \mathrm{H}\right]$ citrulline conversion and a rise in pulmonary cGMP levels from $7 \pm 1$ to $59 \pm 9 \mathrm{pmol} / \mathrm{mg}$ protein in lungs from AdCMVceNOS versus control rats, $(P<0.05)$. During acute hypoxia $\left(\mathrm{FIO}_{2}=0.10\right)$ for $25 \mathrm{~min}$, mean pulmonary artery pressure (PAP) increased significantly from $17 \pm 1$ to $27 \pm 1 \mathrm{mmHg}$ in rats aerosolized with saline $(n=4)$ and from $18 \pm 1$ to $28 \pm 1 \mathrm{mmHg}$ in rats given an adenoviral vector expressing a nuclear-targeted $\beta$-galactosidase gene (AdCMVßgal, $n=8$ ). In contrast, in AdCMVceNOS-transduced rats $(n=8)$ the hypoxia-induced increase in PAP was significantly attenuated $(18 \pm 1$ to $23 \pm 2 \mathrm{mmHg})$. Systemic blood pressure was not affected by aerosol gene transfer. Thus, adenoviral-mediated ceNOS gene transfer to rat lungs increases ceNOS expression and activity, and reduces acute hypoxic pulmonary vasoconstriction. Aerosolized recombinant adenovirus overexpressing vasodilatory proteins can act as a selective pulmonary vasodilator and may hold promise as a future therapeutic strategy for pulmonary hypertension. (J. Clin. Invest. 1996. 98:317-324.) Key words: adenovirus - guanylate cyclase $\cdot$ hypoxia $\cdot$ pulmonary hypertension • gene therapy
\end{abstract}

\section{Introduction}

The pulmonary endothelium regulates pulmonary blood flow and maintains a low vascular resistance through the release of

Address correspondence to Stefan Janssens, M.D., Ph.D., Center for Transgene Technology and Gene Therapy, KU-Leuven, Campus Gasthuisberg, 49 Herestraat, B-3000 Leuven, Belgium. Phone: 16345783; FAX: 16-345990.

Received for publication 27 December 1995 and accepted in revised form 3 May 1996.

J. Clin. Invest.

(C) The American Society for Clinical Investigation, Inc.

0021-9738/96/07/0317/08 \$2.00

Volume 98, Number 2, July 1996, 317-324 vasoactive substances which control vasomotor tone, vascular patency, and normal vessel wall architecture (1). One of these vasoactive substances is nitric oxide (NO), which is synthesized from L-arginine by enzymes called NO synthases (NOSs) (2). There are two classes of NOSs, constitutive and inducible (3). The constitutive enzymes are calcium- and calmodulindependent and were initially identified in brain (NOS I) and endothelial cells (NOS III or ceNOS). The inducible NOS isoform (NOS II or iNOS) is typically expressed in cells only after exposure to cytokines and is calcium-independent $(4,5)$. In vascular smooth muscle cells and in platelets, NO activates soluble guanylate cyclase, which increases intracellular guanosine 3', 5'-cyclic monophosphate (cGMP), thereby inducing vasorelaxation and inhibiting platelet aggregation. NO is thought to be an important modulator in pulmonary hypertension by virtue of its antiplatelet effect and its vasodilatory and antiproliferative actions on pulmonary vascular smooth muscle cells (6-12).

Several observations in vitro and in vivo suggest an important role for NO in the pulmonary vascular response to hypoxia. In isolated-perfused lungs, hypoxia induces a significant reduction in contractile responses to acetylcholine and to inhibitors of NOS (13), and in isolated pulmonary vascular rings hypoxia suppresses basal and agonist-stimulated release of NO $(14,15)$. In endothelial cells, hypoxia inhibits NO production by reducing ceNOS gene transcription and mRNA stability (16). In the lungs of patients with pulmonary hypertension, ceNOS mRNA and protein levels are decreased and correlate inversely with the severity of the plexogenic pulmonary arteriopathy (17). Thus, hypoxia-induced pulmonary hypertension appears to be associated with reduced NO generation from pulmonary endothelium resulting in an imbalance between pulmonary vasoconstrictive and vasodilatory stimuli.

Gene transfer into lungs to restore deficient pulmonary NOS gene expression or to overexpress the biologically active gene product may constitute a new approach to alleviate hypoxic pulmonary hypertension, without systemic hypotensive side effects often associated with pharmacological vasodilator therapy. In rodent lungs, expression of transgenes, either complexed to cationic liposomes or packaged in viral vectors, is dependent on the transfer technique (instillation versus aerosolization) and the vector system used (18-20). Nonviral vectors have a relatively low in vivo transduction efficiency, whereas retroviruses require proliferation of target cells for expression of the newly transferred gene (21). Tracheal instillation of replication-deficient adenoviruses, on the other hand, show diffuse transduction of epithelial cells in proximal bronchi and bronchioles (18). In the present study, we aerosolized recombinant adenoviruses carrying the human ceNOS gene into rat lungs during mechanical ventilation, to obtain diffuse peripheral distribution of the transgene. After gene transfer, the expression of biologically active recombinant ceNOS pro- 
tein and its effect on pulmonary vasoconstriction in acutely hypoxic rats were studied.

\section{Methods}

\section{Construction and purification of recombinant adenovirus}

A 3.7-kilobase pair EcoRI/BamHI fragment of the human endothelial nitric oxide synthase (ceNOS) (22) cDNA was constructed by ligating a 3.4-kb EcoRI/NcoI fragment to a 0.3-kb PCR fragment encoding the carboxyl terminus of the enzyme. The latter was amplified in a polymerase chain reaction using a $5^{\prime}$-CGGCGATGTTACCATGGCAACCAACGT-3' primer encoding amino acid residues 1115-1123 and corresponding to the NcoI site at position 3398 and a $5^{\prime}$-CGGATCCCGGCTCTCAGGGGCTGTTGGTG-3' primer encoding amino acid residues 1199-1203 and including an additional BamHI site at the 3 '-end of the cDNA. For recombinant virus construction, the 3.7-kb fragment comprising the entire protein coding region for ceNOS was cloned between the strong enhancer/promoter of the cytomegalovirus (CMV) immediate early genes and the SV40 polyadenylation signal of the bacterial plasmid pACCMVpLpA (23). The plasmid also contains E1A-deleted sequences of type 5 adenovirus including the origin of replication and the packaging signal and the pUC19 polylinker. Recombinant adenovirus was generated through homologous recombination with pJM17, a bacterial plasmid containing the full-length adenoviral genome, following cotransfection in E1Atransformed human embryonic kidney (293) cells.

The presence of the human ceNOS cDNA insert in virion DNA isolated from infected 293 cells was confirmed by PCR analysis using the 5'-CGGCGATGTTACCATGGCAACCAACGT-3' primer encoding the ceNOS $3^{\prime}$-NADPH-adenine site (amino acid residues 1115-1123) and the 5'-CTCTGTAGGTAGT-TTGTCCA-3' primer complementary to sequences in the polyadenylation signal sequences of pACCMVpLpA. ceNOS-containing viral isolates (AdCMVceNOS) were amplified on confluent 293 cells and, after appearance of cytopathic effects, were isolated, precipitated, and concentrated by discontinuous $\mathrm{CsCl}$ gradient (24). Viral titers were determined by infection of monolayers of 293 cells with serial dilutions of the recombinant adenovirus. Recombinant adenovirus carrying the LacZ gene encoding a nuclear-localizing variant of the E. Coli $\beta$-galactosidase (AdCMVßgal), was amplified and titered as for AdCMVceNOS (25). For all in vivo studies, viral titers were adjusted to $5 \times 10^{9}$ plaque forming units (pfu)/ml. For in vitro studies, a similar viral construct containing a cDNA encoding the thrombin inhibitor hirudin (AdCMVHirudin) was used as a control virus.

\section{Adenovirus-mediated ceNOS gene transduction in vitro}

Detection of ceNOS protein in transduced rat fetal lung fibroblasts. Rat fetal lung fibroblasts (RFL-6) were cultured in DMEM supplemented with $10 \%$ fetal bovine serum (GIBCO BRL, Gaithersburg, $\mathrm{MD}), 50 \mathrm{U} / \mathrm{ml}$ penicillin and $50 \mu \mathrm{g} / \mathrm{ml}$ streptomycin. The cells were grown in chamber slides (Nunc, Naperville, IL) to $\sim 60 \%$ confluence and infected with AdCMVceNOS and AdCMVHirudin diluted in DMEM with $2 \%$ fetal bovine serum at 10 and 100 pfu per cell. After $12 \mathrm{~h}$, the viral suspension was removed, and the cells were maintained in culture for $3 \mathrm{~d}$. The presence of the ceNOS gene product was detected by immunostaining. The third day after in vitro infection with the adenoviral vectors, the cells were washed with phosphate-buffered saline, fixed for $20 \mathrm{~min}$ in $4 \%$ paraformaldehyde and washed twice in $1 \mathrm{mM}$ Tris, $0.9 \% \mathrm{NaCl}, 0.1 \%$ Triton X-100, pH 7.6 (Tris-buffered saline, TBS). Cells were preincubated with swine serum (dilution 1:5) in TBS for $45 \mathrm{~min}$ and exposed overnight to anti-ceNOS pAB $(2 \mathrm{mg} / \mathrm{ml})$, a rabbit polyclonal antibody that recognizes human ceNOS (Transduction Laboratories, Exeter, UK). After $1 \mathrm{~h}$ incubation with a horseradish peroxidase-labeled swine anti-rabbit second antibody (Prosan; diluted 1:50, and preabsorbed overnight with 10\% rat serum and $3 \%$ bovine serum albumin), antibody binding was visualized with diaminobenzidine tetrahydrochloride in $0.1 \mathrm{M}$ Tris buffer, $\mathrm{pH} 7.2$, containing $0.01 \% \mathrm{H}_{2} \mathrm{O}_{2}$. Harris' hematoxylin was used as counterstain and slides were dehydrated and mounted with dePex mountant medium (Prosan, Gent, Belgium).

cGMP levels in transduced RFL- 6 cells. RFL- 6 cells, which contain abundant soluble guanylate cyclase, were grown to $90 \%$ confluence in 12-well tissue culture plates $\left(10^{5}\right.$ cells/well $)$ and infected for $4 \mathrm{~h}$ with either AdCMVceNOS or AdCMVHirudin at 100 pfu per cell, or medium only (DMEM with $2 \%$ fetal bovine serum). Following infection, cells were cultured for $3 \mathrm{~d}$ in DMEM with $10 \%$ fetal bovine serum. Cells were pretreated for $10 \mathrm{~min}$ with $0.3 \mathrm{mM} 3$-isobutyl1-methylxanthine (IBMX, a phosphodiesterase inhibitor) and $2 \mu \mathrm{M}$ A23187 (to stimulate ceNOS activity) in the presence or absence of $0.5 \mathrm{mM} N^{\mathrm{G}}$-nitro-l-arginine methyl ester (L-NAME, a NOS inhibitor). Additional cells were incubated for five minutes with $1 \mathrm{mM}$ sodium nitroprusside (a NO-donor compound), as positive control. Intracellular cGMP was extracted in ice-cold $15 \%$ trichloroacetic acid (TCA), $\mathrm{pH}$ 4.0. TCA was extracted in $\mathrm{H}_{2} \mathrm{O}$-saturated ether and, following lyophilization, cGMP was quantitated using a commercial enzyme-immunoassay (Amersham Life Science, Ghent, Belgium).

\section{Adenovirus-mediated ceNOS gene transduction in vivo}

Gene delivery. Wistar rats (300-350 grams body weight) were anesthetized by intraperitoneal injection of pentobarbital $(50 \mathrm{mg} / \mathrm{kg})$, incubated with a polyethylene tube (No. PE-240 tubing; $1.67 \mathrm{~mm}$ ID), and mechanically ventilated with room air (Model 683; Harvard apparatus, South Natick, MA). During mechanical ventilation, $600 \mu \mathrm{l}$ solution of recombinant adenovirus (AdCMVßgal or AdCMVceNOS, $5 \times 10^{9} \mathrm{pfu} / \mathrm{ml}$ ) was aerosolized into the lungs via a silastic catheter introduced via a midline neck incision into the trachea distal from the endotracheal tube. Using a tuberculine syringe, a total volume of $600 \mu \mathrm{l}$ viral solution was administered drop by drop during the inspiratory phase of the ventilatory cycle $(50 \mu \mathrm{l} / 10 \mathrm{~min})$. Tidal volume was set at $2.5 \mathrm{ml}$, and frequency at $60 / \mathrm{min}$. After viral delivery, the catheter was removed from the trachea. Control rats were given an equal volume of sterile saline solution. No side effects were observed during aerosol delivery or after extubation.

Tissue distribution of aerosolized recombinant adenovirus. $4 \mathrm{~d}$ after aerosolization of AdCMV $\beta$ gal into the lungs, animals were killed by administration of an overdose pentobarbital, and the lungs were perfused and fixed through the airways in $4 \%$ (wt/vol) formaldehyde. Two mm thick segments from the central and peripheral areas of all lobes were incubated in $20 \%$ sucrose overnight, overlaid with O.C.T compound and frozen in liquid nitrogen. Seven $\mu \mathrm{m}$ cryostat sections were mounted on poly-l-lysine coated slides, and the presence and distribution of the LacZ gene was detected using $\beta$-galactosidase staining [ $5 \mathrm{mmol} / \mathrm{liter} \mathrm{K}_{4} \mathrm{Fe}(\mathrm{CN})_{6}, 5 \mathrm{mmol} / \mathrm{liter} \mathrm{K}_{3} \mathrm{Fe}(\mathrm{CN})_{6}, 1 \mathrm{mmol} / \mathrm{li}$ ter $\mathrm{MgCl}_{2}$ and $1 \mathrm{mg} / \mathrm{ml}$ 5-bromo-4-chloro-3-indolyl- $\beta$-D-galactopyranoside in PBS] for 4-6 h, and counterstaining with eosin. To estimate gene transfer efficiency in the histological sections, positive cells were identified by the blue coloration of their nuclei.

Measurements of ceNOS protein levels. Expression of ceNOS in rat lungs was assessed on day 4 after gene transfer. Animals were killed, and the lungs were excised and processed immediately or quick frozen in liquid nitrogen. To extract total protein, lungs were homogenized in ice cold buffer (Hepes $5 \mathrm{mM}$, pH 7.9, glycerol $26 \%$ (vol/vol), $\mathrm{MgCl}_{2} 1.5 \mathrm{mM}$, EDTA $0.2 \mathrm{mM}$, DTT $0.5 \mathrm{mM}$, phenylmethylsulfonyl fluoride $0.5 \mathrm{mM}$ ), with $\mathrm{NaCl}$ (300 $\mathrm{mM}$ final), and incubated on ice for $30 \mathrm{~min}$. After centrifugation at $100,000 \mathrm{~g}$ at $4^{\circ} \mathrm{C}$ for $20 \mathrm{~min}$, the supernatant containing crude enzyme preparations was mixed with an equal volume $2 \% \mathrm{SDS} / 1 \% \beta$ mercaptoethanol and fractionated using $8 \%$ SDS/PAGE ( $70 \mu \mathrm{g}$ per lane). Proteins were then transferred to a nitrocellulose membrane (Hybond-ECL; Amersham Life Sciences, Gent, Belgium) by semi-dry electroblotting for $1 \mathrm{~h}$. The membranes were blocked $1 \mathrm{~h}$ at room temperature with blotto-Tween (5\% nonfat dry milk, $0.1 \%$ Tween-20) and incubated with a primary monoclonal mouse anti-ceNOS IgG1 antibody (mAb, $0.25 \mathrm{mg} / \mathrm{ml}$, dilution 1:1000; Transduction Laboratories, Exeter, UK). Bound antibody was detected with horseradish peroxidase-labeled rabbit antimouse IgG second antibody (Prosan, dilution 1:2000 in blotto/Tween) 
and visualized using enhanced chemiluminescence (ECL, Amersham, Ghent, Belgium).

Localization of ceNOS immunoreactivity. Lungs were perfused through the pulmonary artery with PBS, and $4 \%$ formaldehyde was instilled into the airways. Lungs were divided in small central and peripheral segments corresponding to the different lobes, and the segments were overlaid with O.C.T compound and frozen in liquid nitrogen. Seven $\mu \mathrm{m}$ cryostat sections were mounted on slides, washed twice with TBS and blocked with normal rabbit serum, diluted 1:5 in TBS, for $45 \mathrm{~min}$. The sections were incubated overnight with the same anti-ceNOS antibody $(2 \mathrm{mg} / \mathrm{ml})$ followed by incubation for $1 \mathrm{~h}$ with a rabbit anti-mouse $\operatorname{IgG}$ peroxidase conjugate (dilution 1:50; preabsorbed overnight at $4^{\circ} \mathrm{C}$ with $10 \%$ preimmune rat serum and $3 \%$ bovine serum albumin). Antibody binding was visualized with 3,3-diaminobenzidine tetrahydrochloride (DAB, Sigma Chemical Co.) in $0.1 \mathrm{M}$ Tris buffer, $\mathrm{pH} 7.2$, containing $0.01 \% \mathrm{H}_{2} \mathrm{O}_{2}$. Sections were counterstained with Harris' hematoxylin, dehydrated, and mounted with dePex mountant medium.

Measurement of pulmonary ceNOS enzyme activity. Tomeasure ceNOS enzyme activity, L-arginine to L-citrulline conversion was assayed in pulmonary extracts using a modification of the method described by Xue et al. (26). Lung protein extracts prepared as described above were purified by affinity chromatography on $2^{\prime}$, $3^{\prime}$-ADP Sepharose and incubated for $30 \mathrm{~min}$ at $37^{\circ} \mathrm{C}$ in a solution of $10 \mathrm{mM} \mathrm{L}-\left[2,3-{ }^{3} \mathrm{H}\right]$ arginine $(59 \mathrm{Ci} / \mathrm{mmol}: 1 \mathrm{Ci}=37 \mathrm{Gbq}), 1 \mathrm{mM}$ NADPH, $100 \mathrm{nM}$ calmodulin, $2 \mathrm{mM} \mathrm{CaCl} 2,20 \mu \mathrm{M}$ tetrahydrobiopterin in a final volume of $1 \mathrm{ml}$. To inhibit NOS activity, duplicate samples were incubated in the presence of $0.5 \mathrm{mM} N^{\mathrm{G}}$-nitro-l-arginine methyl ester (L-NAME). The reaction was stopped by adding $1 \mathrm{ml}$ of stop buffer ( $2 \mathrm{mM}$ EGTA/2 mM EDTA/20 mM Hepes buffer, $\mathrm{pH}$ $5.5)$ to $200 \mu \mathrm{l}$ aliquots of the reaction mixture. The total volume was then applied to a 1-ml Dowex AG 50WX-8 column $\left(\mathrm{Na}^{+}\right.$form, BioRad Laboratories, Nazareth Eke, Belgium) preequilibrated with the stop buffer. L-[2,3- $\left.{ }^{3} \mathrm{H}\right]$ citrulline was eluted with $2 \mathrm{ml}$ of distilled water and the radioactivity was measured by liquid scintillation counting. Enzyme activity was expressed as citrulline production in pmol.min ${ }^{-1} \cdot \mathrm{mg} \mathrm{protein}^{-1}$.

Measurement of pulmonary cGMP levels. For cGMP determinations, lungs were frozen in liquid nitrogen and 400-700 mg tissue samples were subsequently homogenized in $1 \mathrm{ml}$ ice-cold $6 \%$ trichloroacetic acid (TCA), $\mathrm{pH}$ 4.0. Each sample was then centrifuged at $10,000 \times g$ for $15 \mathrm{~min}$ at $4^{\circ} \mathrm{C}$. The supernatant was transferred into a 30 -ml glass centrifuge tube, and TCA was extracted with $\mathrm{H}_{2} \mathrm{O}$-saturated ether four times. A 500- $\mu$ l aliquot of the sample was then lyophilized, resuspended in $500 \mu \mathrm{l}$ of $0.05 \mathrm{M}$ sodium acetate buffer $(\mathrm{pH}$ 5.8) and assayed for cGMP using a nonradioactive enzyme-immunoassay kit (Amersham Life Science). Pulmonary cGMP levels were described as picomoles cGMP per mg of TCA-precipitable protein.

\section{Measurement of the pulmonary vasoconstrictor response to hypoxia}

After recovery from the aerosol gene delivery procedure, rats were maintained in room air for three days, and reanesthetized on the fourth day after gene transfer. To measure pulmonary artery pressure (PAP), a silastic catheter $(0.30 \mathrm{~mm} \mathrm{ID}, 0.64 \mathrm{~mm}$ OD) was introduced into the right jugular vein and advanced through the right atrium and right ventricle into the pulmonary artery. To measure systemic arterial pressure, a $2 \mathrm{~F}$ catheter was positioned in the right carotid artery. Both carotid arterial and pulmonary artery catheters were connected to a pressure transducer (Model AA 016; Baxter, Uden, Holland), a display oscilloscope (Press Ampl. 863, Siemens, Germany) and a thermal recorder (Mingograf 82, Siemens, Solna, Sweden). The position of the pulmonary artery catheter was verified by the characteristic pressure tracing on the oscilloscope and was confirmed by autopsy. Electronically meaned PAP and right ventricular systolic pressures were recorded. Cardiac output was measured by the thermodilution technique which was validated in rodents in previous studies (27). Briefly, a $1.5 \mathrm{~F}$ thermodilution probe was inserted in the thoracic aorta via the right carotid artery and connected to a thermal dilution computer (model REF-1, Baxter Edwards, Irvine, CA), and a strip-chart recorder. Through the silastic pulmonary artery catheter, $0.15 \mathrm{ml}$ saline was injected and cardiac output was read directly from the computer display. All values were measured in triplicate and varied $<15 \%$. Cardiac index $(\mathrm{CI})$ was defined as the ratio of cardiac output over body weight in kilograms $\left(\mathrm{ml} \cdot \mathrm{min}^{-1} \cdot \mathrm{kg}^{-1}\right)$. Total pulmonary vascular resistance index (TPRI) was computed by dividing mean pulmonary artery pressure by cardiac index $\left(\mathrm{mmHg} \cdot \mathrm{min}^{-1} \cdot \mathrm{ml}^{-1} \cdot \mathrm{kg}^{-1}\right)$.

Rats were initially mechanically ventilated with room air and baseline PAP, systemic blood pressure, and cardiac output were recorded. To study the hypoxic pulmonary vasoconstrictor response, rats were ventilated with $10 \% \mathrm{O}_{2}$ and $90 \% \mathrm{~N}_{2}$, and PAP was monitored continuously for $25 \mathrm{~min}$. After 5, 15, and $25 \mathrm{~min}$ of hypoxia, cardiac output was measured. Rats were then ventilated with room air and hemodynamic measurements were repeated after $20 \mathrm{~min}$.

\section{Statistical analysis}

Analysis of variance (ANOVA) followed by the Student-NeumannKeuls post test was used to determine significant differences in multiple comparison testing between groups. All values are expressed as means \pm SEM. For all experiments, statistical significance was assumed at $P<0.05$.

\section{Results}

Transduction of rat fetal lung fibroblasts with AdCMVceNOS. To determine whether high levels of recombinant ceNOS protein could be achieved in cultured cells using adenovirus-mediated gene transfer, rat fetal lung fibroblasts (RFL6cells) were infected with AdCMVceNOS, fixed, and stained with a polyclonal anti-ceNOS antibody. Multiplicities of infection (MOI) of 10 and 100 were selected because a higher MOI was associated with cytopathic effects. After $3 \mathrm{~d}$, abundant ceNOS was observed in the AdCMVceNOS-infected cells but not in the AdCMVHirudin-infected cells (Fig. 1). ceNOS expression was detectable after $24 \mathrm{~h}$, and the percentage of ceNOS-positive cells peaked at $3 \mathrm{~d}$ after infection (data not shown).

To investigate whether the transduced ceNOS gene encodes a biologically active protein capable of producing NO, RFL-6 cells containing abundant soluble guanylate cyclase were infected at a MOI of 100 with AdCMVceNOS or AdCMVHirudin. Intracellular cGMP was measured in cells exposed to the calcium ionophore A23186 (to activate ceNOS) and IBMX (to inhibit phosphodiesterases). cGMP levels did not differ between uninfected RFL-6 cells and RFL-6 cells infected with AdCMVHirudin. cGMP levels were markedly increased in RFL-6 cells infected with AdCMVceNOS, and in cells exposed to sodium nitroprusside (SNP), a NO donor compound (Fig. 2). Preincubation of RFL-6 cells with $0.5 \mathrm{mM}$ L-NAME for 30 min markedly reduced cGMP levels in AdCMVceNOS-infected cells. The L-NAME-inhibitable increase in cGMP levels in AdCMVceNOS-infected RFL- 6 cells suggested that the transgene encoded a biologically active NOS.

Transduction of lung tissue after aerosolization of recombinant AdCMVBgal and AdCMVceNOS. To achieve high levels of transgene expression in peripheral pulmonary tissues, recombinant adenoviruses were aerosolized in vivo in rat lungs during mechanical ventilation. Recombinant adenovirus carrying the $L a c Z$ gene was used to study the distribution of transgene expression. AdCMVßgal-infected lungs showed diffuse transduction of airway epithelial cells in proximal bronchi and distal bronchioles, alveolar lining cells, and adventitial cells in medium- and small-sized pulmonary vessels as demonstrated by $\beta$-galactosidase staining after 5 days (Fig. $3 A$ ). 

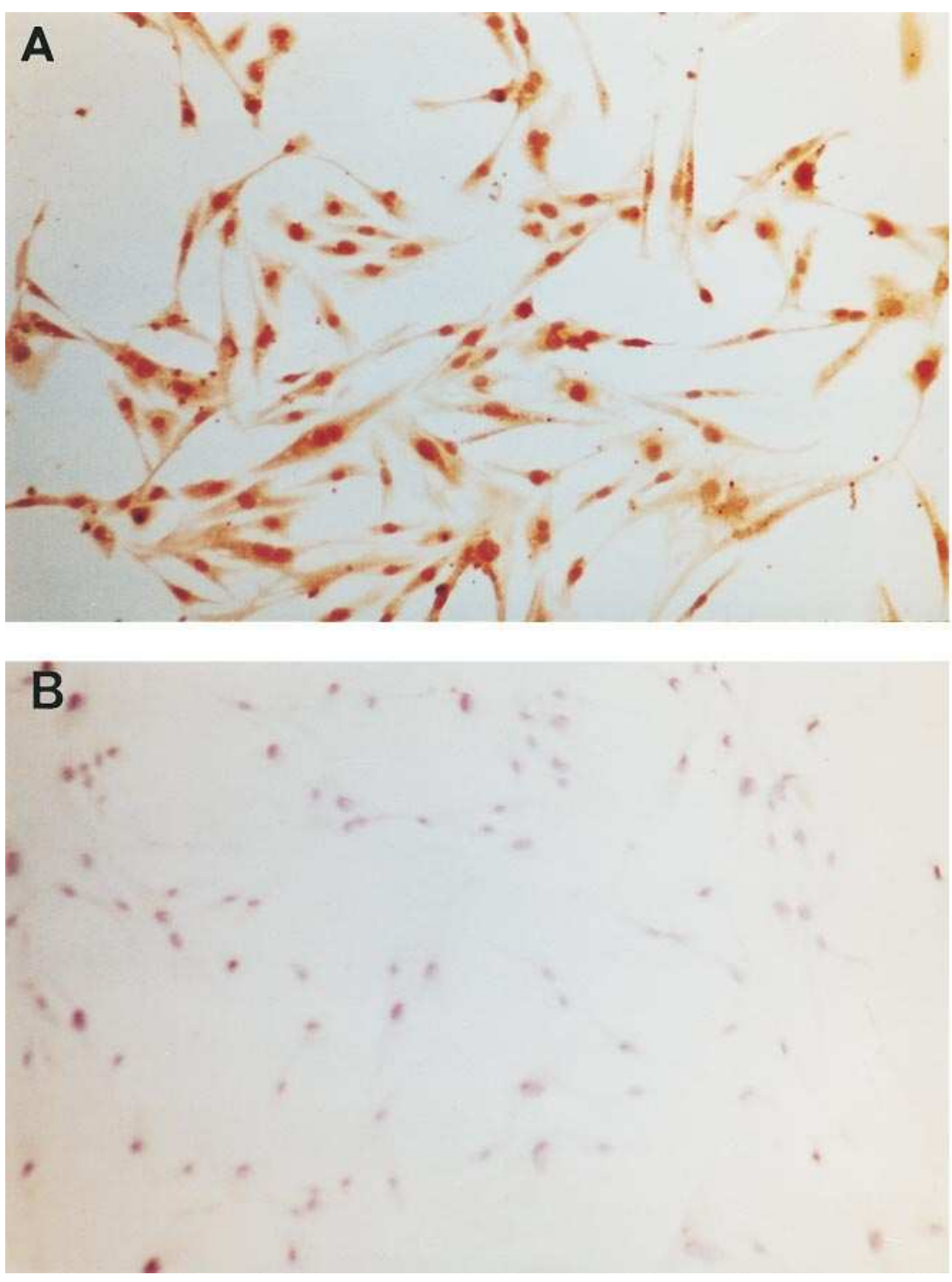

Figure 1. Immunostaining for ceNOS in cultured rat fetal lung fibroblasts. Abundant ceNOS immunoreactivity is observed in AdCMVceNOS-infected $(A, t o p)$ but not in AdCMVHirudin-infected ( $B$, bottom) cells $(\times 200)$.

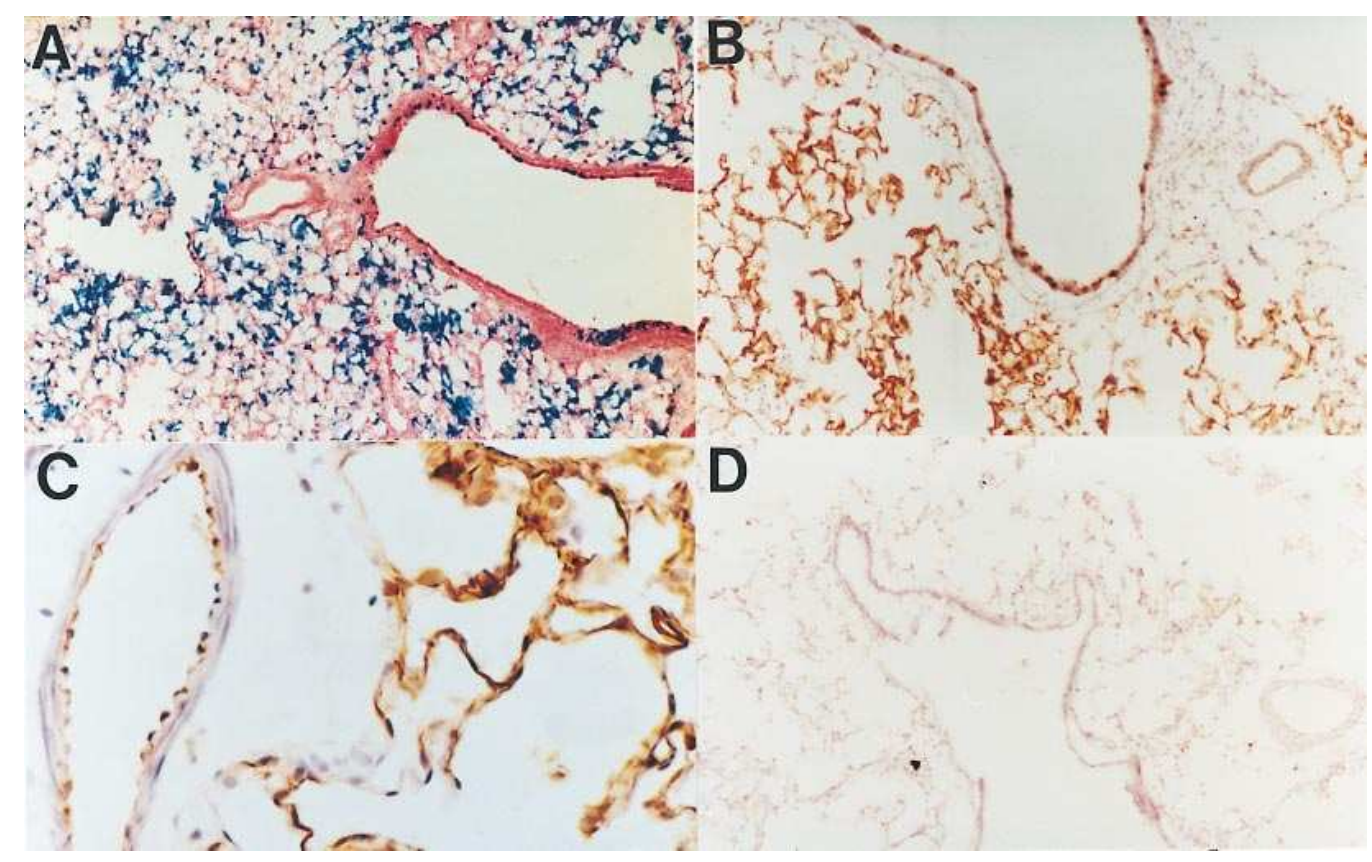

Figure 3. ceNOS and Bgalactosidase gene expression in lungs of rats transduced with AdCMVceNOS and AdCMVßgal. AdCMV $\beta$ gal transduced rat lungs show nuclear localized $\beta$-galactosidase staining in basal airway epithelial cells, alveolar epithelial cells and adventitial cells of small pulmonary vessels $(A, \times 200)$. ceNOS was observed in bronchial and alveolar epithelial cells, and in the endothelium of medium-sized and small pulmonary vessels of AdCMVceNOS infected rats $(B$, $\times 200$, and $C, \times 400$ ), but not in AdCMV $\beta$ gal treated rats $(D, \times 200)$. 


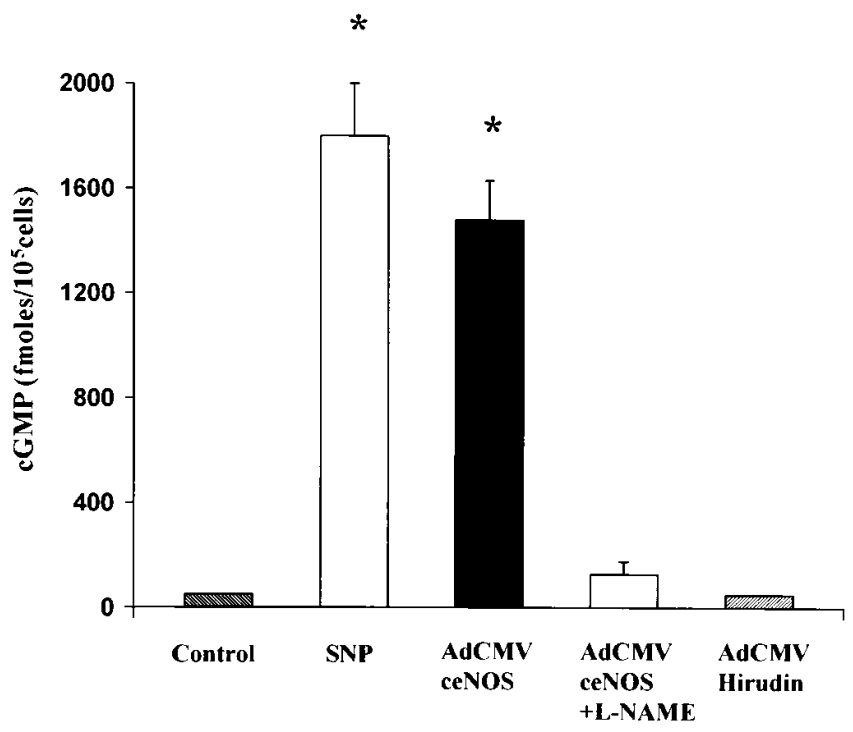

Figure 2. Cyclic GMP production in RFL-6 cells. Cellular cGMP content was measured under baseline conditions, and after infection with AdCMVceNOS in the absence and presence of L-NAME. Sodium nitroprusside (SNP) was used as positive control, and AdCMVHirudin, expressing the thrombin inhibitor hirudin was used as control virus carrying an unrelated gene. Data are means \pm SEM of five determinations, except for AdCMVHirudin. $* P<0.05$ vs. Control,

AdCMVceNOS+L-NAME, and AdCMVHirudin.

The intensity and distribution of ceNOS expression in the lungs after aerosolization of AdCMVceNOS was studied at various times $(3,4,5,8$, and $12 \mathrm{~d})$ after gene transfer by immunostaining with monoclonal antibodies directed against human ceNOS. ceNOS immunostaining was observed in the majority of epithelial cells of the large airways including ciliated cells, in alveolar lining cells of the lung parenchyma, and in endothelial cells of medium-sized and small pulmonary vessels (Fig. 3, B and $C$ ). The intensity of staining was maximal after $5 \mathrm{~d}$ but was still detected after 2 wk (not shown). In control and AdCMVßgal-treated rats, no ceNOS immunoreactivity was detected in large airways, alveolar epitelial cells or in small pulmonary vessels (Fig. 3 D). Endogenous ceNOS immunoreactivity was only detected in endothelial cells of large, fully muscular vessels (data not shown). There was little variation in the staining pattern among animals infected with AdCMVceNOS. Infection with a titer of $5 \times 10^{9} \mathrm{pfu} / \mathrm{ml}$ AdCMVceNOS was not associated with any significant pulmonary infiltrates and did not affect body weights.

Cytokines released during local inflammatory reactions or in response to adenoviral infection could activate the inducible isoform of NOS (iNOS). We investigated whether gene transfer and the associated immune response against adenovirus would stimulate iNOS gene expression in rat lungs. No iNOS immunoreactivity was observed using a specific anti-iNOS antiserum (Transduction Laboratories) on sections from AdCMVceNOS- or AdCMVßgal-treated rats (data not shown). These results suggest that recombinant adenoviral infection itself does not stimulate NOS production via inflammation and induction of the inducible isoform of NOS.

ceNOS protein levels in adenoviral-infected and control lungs were measured by immunoblot analysis of extracts from

control, AdCMVßgal-, and AdCMVceNOS-treated rat lungs. A ceNOS-specific monoclonal antibody detected abundant levels of the $135-\mathrm{kD}$ protein in the lung extracts of rats $4 \mathrm{~d}$ after treatment with AdCMVceNOS (Fig. 4). Only very low levels were detected in lung extracts of AdCMVßgal-infected rats, or in lung extracts of untreated control rats.

To measure ceNOS enzyme activity, $\left[{ }^{3} \mathrm{H}\right] \mathrm{L}$-arginine to $\left[{ }^{3} \mathrm{H}\right] \mathrm{L}$-citrulline conversion was measured in extracts from AdCMVßgal- and AdCMVceNOS-transduced lungs. $\left[{ }^{3} \mathrm{H}\right] \mathrm{L}-$ citrulline formation was $86 \%$ greater in lung extracts from AdCMVceNOS-treated rats compared to AdCMVßgal-treated animals, and this increased conversion was blocked in the presence of L-NAME. Simultaneously, biological activity of the expressed ceNOS was evaluated by measuring intrapulmonary cGMP. cGMP levels were $\sim 10$-fold greater in AdCMVceNOS-transduced lungs compared to control lungs and AdCMVßgal-transduced lungs $(59 \pm 9 \mathrm{pmol} / \mathrm{mg}$ protein vs $7 \pm 1$ and $3 \pm 1 \mathrm{pmol} / \mathrm{mg}$ protein respectively, $P<0.05)$.

Aerosolization of recombinant AdCMVceNOS attenuates hypoxia-induced pulmonary vasoconstriction. To investigate whether increased production of $\mathrm{NO}$ following AdCMVceNOS gene transfer would decrease hypoxic pulmonary vasoconstriction, rats were exposed to $10 \%$ oxygen for $25 \mathrm{~min}$. PAP measured in room air was similar in all groups $(17 \pm 1$ $\mathrm{mmHg}$ in saline control, and $18 \pm 1 \mathrm{mmHg}$ in AdCMVßgaland AdCMVceNOS-transduced rats, $P=$ NS). During acute hypoxia, PAP increased from $17 \pm 1$ to $27 \pm 1 \mathrm{mmHg}$ and from $18 \pm 1$ to $28 \pm 1 \mathrm{mmHg}$ in saline control and AdCMV $\beta$ gal-transduced rats, respectively. In contrast, the rise in PAP was markedly attenuated in AdCMVceNOS-transduced rats $(23 \pm 2$ $\mathrm{mmHg}, P<0.05$, Fig. 5, left) and was significantly decreased as early as $5 \mathrm{~min}$ of hypoxia. The effect persisted for the entire duration of the acute challenge, even when sustained for more than $2 \mathrm{~h}$ (data not shown). Cardiac index did not differ between AdCMVceNOS-treated rats and AdCMVßgal-infected

1

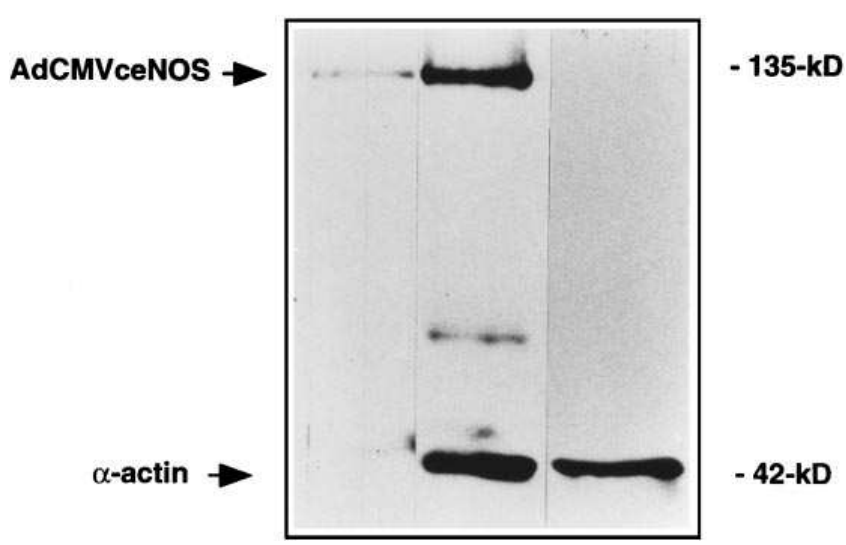

Figure 4. Expression of ceNOS in rat lungs. Protein extracts were prepared from the lungs of rats aerosolized with AdCMVceNOS (lane 2) and AdCMVßgal (lane 3). Samples ( $70 \mu \mathrm{g}$ ) were fractionated using SDS-PAGE and transferred to nitrocellulose membranes. ceNOS (135 kD) was detected using a monoclonal antibody. A ceNOS standard from human umbilical vein endothelial cells, supplied by the manufacturer, was used as a positive control (lane 1). A monoclonal antibody directed against alpha-actin $(42 \mathrm{kD})$ was used to control for possible sample variability. 


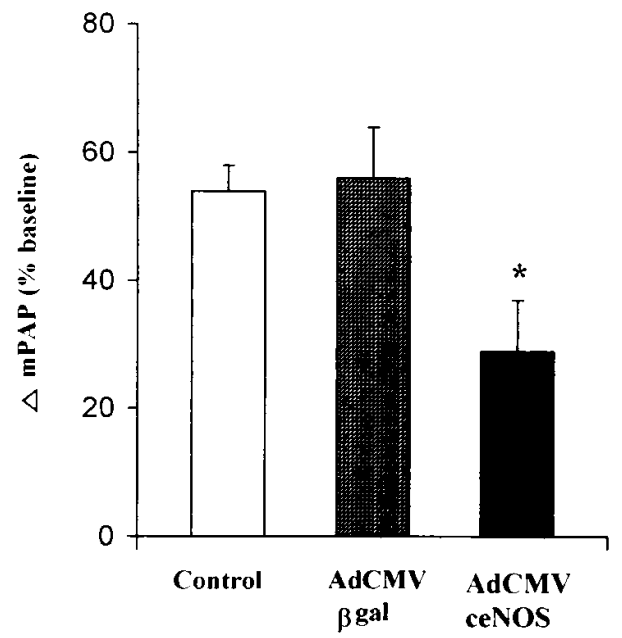

rats $(185 \pm 15 \mathrm{ml} / \mathrm{min} / \mathrm{kg}$ vs $176 \pm 18 \mathrm{ml} / \mathrm{min} / \mathrm{kg}$, respectively). The reduction in total pulmonary resistance index (TPRI) during acute hypoxia in AdCMVceNOS-treated rats compared with AdCMVßgal-infected rats $\left(0.157\right.$ to $0.122 \mathrm{mmHg} \cdot \mathrm{ml}^{-1}$. $\left.\min ^{-1} \cdot \mathrm{kg}^{-1}, P<0.05\right)$ was therefore due to a direct pulmonary vasodilatory effect rather than an indirect effect on cardiac output (Fig. 5, right). Systemic blood pressure was similar in

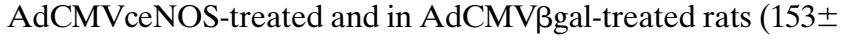
$6 \mathrm{mmHg}$ vs $150 \pm 6 \mathrm{mmHg}$, respectively, $n=4)$. Taken together, aerosolization of AdCMVceNOS and the resulting overexpression of the ceNOS gene product significantly reduced acute hypoxic vasoconstriction without affecting systemic blood pressure or cardiac index.

\section{Discussion}

NO-dependent vasodilatation plays an important role in the adaptation of pulmonary vascular resistance to changes in oxygen tension. In the present study, recombinant adenovirus carrying the gene encoding human constitutive endothelial NOS (ceNOS) was aerosolized into rat lungs and its effect on acute hypoxic pulmonary hypertension was measured. After AdCMVceNOS gene transfer, immunostaining of lung sections with an isoform-specific monoclonal antibody localized ceNOS immunoreactivity in basal airway epithelial cells, lung alveolar lining cells, and adventitial and endothelial cells of both mediumsized and small pulmonary vessels (Fig. 3). The immunohistochemical findings were paralleled by abundant levels of immunoreactive ceNOS protein in lung extracts from AdCMVceNOS aerosolized rats (Fig. 4). Extracts from untransduced control rat lungs displayed low levels of immunoreactivity as described previously (28). Transduced ceNOS produced NO in rat lungs, as evidenced by increased arginine to citrulline conversion by lung extracts and increased pulmonary cGMP levels. L-arginine to L-citrulline conversion in extracts from AdCMVceNOS was blocked by L-NAME suggestive of a NOS specific catalytic reaction. Aerosolization of AdCMVceNOS
Figure 5. Hypoxic pulmonary vasoreactivity in AdCMVceNOS vs. AdCMVßgal transduced rats. Maximal changes in mean pulmonary artery pressure (mPAP, left) and total pulmonary resistance index (TPRI, right) are shown in uninfected rats (open bars, $n=8$ ), AdCMVßgal transduced rats (dashed bars, $n=8$ ), and AdCMVceNOS transduced rats (closed bars, $n=8$ ) during a $25 \mathrm{~min}$ acute hypoxic challenge. Pressure and resistance changes are expressed as percentage of baseline. $* P<0.05$ vs. controls and AdCMVßgal. Values are means \pm SEM. reduced the rise in pulmonary artery pressure and total pulmonary resistance during acute hypoxia (Fig. 5), without affecting systemic blood pressure suggesting aerosolized recombinant adenovirus containing ceNOS gene acts as a selective pulmonary vasodilator.

The importance of the NO signaling pathway in the pathophysiology of pulmonary hypertension is well recognized (29). Many observations in animals $(30,31)$ and patients $(32-34)$ support the view that endothelial dysfunction or injury contributes to different forms of pulmonary hypertension, including hypoxic pulmonary hypertension. Endothelial dysfunction associated with decreased NO production is likely to play a role in the development of pulmonary hypertension as vasoconstrictive stimuli are no longer counterbalanced by the vasorelaxant NO. Some of the underlying molecular mechanisms in this process have recently been identified. In vitro studies in endothelial cells exposed to low oxygen concentrations revealed that hypoxia inhibited ceNOS gene expression via transcriptional and posttranscriptional mechanisms (16). A recent study in patients with primary and secondary pulmonary hypertension reported also reduced ceNOS expression in pulmonary vascular endothelium. Using immunohistochemistry and in situ hybridization, the lungs of patients with pulmonary hypertension were found to contain significantly reduced ceNOS immunoreactivity and ceNOS messenger RNA and showed a significant inverse correlation between the level of immunoreactivity and the severity of the pathologic alterations in the pulmonary vasculature (17).

We, therefore, sought to enhance ceNOS gene expression via pulmonary gene transfer in rats and to explore its potential therapeutic benefit during hypoxia-induced pulmonary hypertension. Rats showed no signs of respiratory distress either during or following aerosol gene transfer and a single dose did not elicit major intrapulmonary or systemic toxic effects. The delivery technique used in the present study resulted in a more uniform and deeper penetration of the virus than could be achieved with intratracheal instillation during spontaneous 
breathing. A homogeneously-distributed transduction pattern was observed in all animals when a virion titer of $5 \times 10^{9} \mathrm{pfu} /$ $\mathrm{ml}$ was administered. In contrast, virion titers exceeding $1 \times$ $10^{10} \mathrm{pfu} / \mathrm{ml}$ elicited a local peribronchial and alveolar inflammatory reaction (data not shown). We also considered the possibility that in virus-infected lungs the increased NO production might have resulted from expression of the inducible NOS isoform, secondary to inflammation and cytokine activation (35). Several lines of evidence argue against this possibility. First, no increased NO production was found in bioactivity assays on extracts from lungs transduced with control adenovirus. Second, immunohistochemistry using an inducible NOSspecific antibody did not detect intrapulmonary expression of this isoform after viral infection, which is consistent with the absence of a major inflammatory reaction.

Aerosol transfer of recombinant adenovirus carrying the ceNOS gene in rat lungs increased NOS protein expression in the peripheral lung parenchyma surrounding small pulmonary resistance vessels. The observed pulmonary vasodilatory effect may have resulted from recombinant gene expression in or around these vessels with direct stimulation of soluble guanylate cyclase in pulmonary vascular smooth muscle cells by NO. In control experiments using AdCMVßgal, transgene expression was localized not only in airway epithelial cells but also in adventitial cells of small- and medium-sized blood vessels. Alternatively, since gases within alveoli can reach precapillary vessels (36), NO released in the alveolar airspace following transduction of distal lung parenchyma may have readily diffused from the airspace to precapillary resistance vessels and mediated pulmonary vasodilatation.

ceNOS gene transfer thus locally produced NO which selectively vasodilated pulmonary vessels, in a similar fashion as was observed with NO gas therapy in hypoxic newborn rats (8), in lambs (37), and in human volunteers breathing $12 \% \mathrm{O}_{2}$ in $\mathrm{N}_{2}$ (38). Inhaled NO did not affect pulmonary hemodynamics during room air breathing and caused no systemic hemodynamic effects, possibly because any NO which diffused into the blood stream was rapidly inactivated by hemoglobin (39). Similarly, AdCMVceNOS aerosol did not affect systemic blood pressure. However, NO gas inhalation only has an immediate and shortlasting effect on pulmonary hemodynamics, and requires continuous administration (40). By contrast, our study showed that a single aerosol dose of AdCMVceNOS was able to attenuate hypoxic pulmonary vasoconstriction even when the hypoxic challenge was applied 4-7 d after gene delivery. Whether attenuation of the hypoxic vasoconstrictor response would also affect pulmonary vascular remodeling associated with chronic hypoxic pulmonary hypertension remains to be determined. Also, whether local overexpression of a calcium independent NOS isoform has a different effect on hypoxic pulmonary hypertension is so far unknown. The present study shows that adenoviral mediated recombinant ceNOS expression in lungs appears to be safe and could have potential clinical applications as adjunctive treatment in some pulmonary hypertensive diseases, including persistent pulmonary hypertension of the newborn, perioperative pulmonary hypertension, and adult respiratory distress syndrome (41-43).

In summary, delivery of recombinant ceNOS adenovirus by a single aerosolization enables diffuse transduction of bronchial and alveolar epithelial cells and vascular adventitial and endothelial cells and is capable of mediating increased NO production. During acute hypoxia, local overexpression of hu- man ceNOS in rat lungs significantly reduced the rise in pulmonary artery pressure and total pulmonary resistance without adverse effects on systemic hemodynamics. Aerosolized ceNOS gene thus acts as a selective pulmonary vasodilator and this novel approach may represent an example for future gene therapy applications in patients with pulmonary hypertension.

\section{Acknowledgments}

The authors thank Dr. C.A. Hales for his advice, and N. Van Pelt, H. Gillijns, and E. Vertenten for expert technical assistance.

This work was supported by the National Fund for Scientific Research (NFWO), Belgium, and the Belgian Society of Cardiology (to S. Janssens), and by National Institutes of Health grant HL-45895 and by a grant to the Cardiovascular Research Center from Bristol Meyers Squibb Pharmaceuticals (to K.D. Bloch). S. Janssens is the recipient of a chair financed by Zeneca Pharmaceuticals Inc.

\section{References}

1. Vanhoutte, P.M. 1988. The endothelium-modulator of vascular smooth muscle tone. N. Engl. J. Med. 319:512-513.

2. Nathan, C. 1992. Nitric oxide as a secretory signal of mammalian cells. FASEB J. 6:3051-3064.

3. Nathan, C., and Q.W. Xie. 1994. Nitric oxide synthases: roles, tolls, and controls. Cell. 78:915-918.

4. Stuehr, D.J., and O.W. Griffith. 1992. Mammalian nitric oxide synthases. Adv. Enzymol. Relat. Areas Mol. Biol. 65:287-346.

5. Lowenstein, C.J., C.S. Glatt, D. Bredt, and S.H. Snyder. 1992. Cloned and expressed macrophage nitric oxide synthase contrasts with the brain enzyme. Proc. Natl. Acad. Sci. USA. 89:6711-6715.

6. Moncada, S., R.M. Palmer, and E.A. Higgs. 1991. Nitric oxide: physiology, pathophysiology, and pharmacology. Pharmacol. Rev. 43:109-142.

7. Garg, U.C., and A. Hassid. 1989. Nitric oxide-generating vasodilators and 8-bromo-cyclic guanosine monophosphate inhibit mitogenesis and proliferation of cultured rat vascular smooth muscle cells. J. Clin. Invest. 83:1774-1777.

8. Roberts, J.D., C.T. Roberts, R.C. Jones, W.M. Zapol, and K.D. Bloch. 1995. Continuous nitric oxide inhalation reduces pulmonary arterial structural changes, right ventricular hypertrophy, and growth retardation in the hypoxic newborn rat. Circ. Res. 76:215-222.

9. Heath, D. 1993. The pathology of pulmonary hypertension. Eur. Respir. Rev. 3:555-558.

10. Radomski, M.W., R.M.J. Palmer, and S. Moncada. 1987. The role of nitric oxide and cGMP in platelet adhesion to vascular endothelium. Biochem. Biophys. Res. Commun. 148:1482-1489.

11. Assender, J.W., K.M. Southgate, and A.C. Newby. 1991. Does nitric oxide inhibit smooth muscle cell proliferation? J. Cardiovasc. Pharmacol. 17:104107.

12. deGraaf, J.C., J.D. Banga, S. Moncada, R.M.J. Palmer, P.J. deGroot, and J.J. Sixma. 1992. Nitric oxide functions as an inhibitor of platelet adhesion under flow conditions. Circulation. 85:2284-2290.

13. Adnot, S., B. Raffestin, S. Eddahibi, P. Braquet, and P. Chabrier. 1991. Loss of endothelium-dependent relaxant activity in the pulmonary circulation of rats exposed to chronic hypoxia. J. Clin. Invest. 87:155-162.

14. Johns, R.A., J.M. Linden, and M.J. Peach. 1989. Endothelium-dependent relaxation and cyclic GMP accumulation in rabbit pulmonary artery are selectively impaired by moderate hypoxia. Circ. Res. 65:1508-1515.

15. Shaul, P.W., L.B. Wells, and K.M. Horning. 1993. Acute and prolonged hypoxia attenuate endothelial nitric oxide production in rat pulmonary arteries by different mechanisms. J. Cardiovasc. Pharmacol. 22:819-827.

16. McQuillan, L.P., G.K. Leung, P.A. Marsden, S.K. Kostyk, and S. Kourembanas. 1994. Hypoxia inhibits expression of eNOS via transcriptional and posttranscriptional mechanisms. Am. J. Physiol. 267:H1921-H1927.

17. Giaid, A., and D. Saleh. 1995. Reduced expression of endothelial nitric oxide synthase in the lungs of patients with pulmonary hypertension. $N$. Engl. J. Med. 333:214-221.

18. Mastrangeli, A., C. Danel, M. Rosenfeld, L. Stratford-Perricaudet, M. Perricaudet, A. Pavirani, J. Lecocq, and R. Cristal. 1993. Diversity of airway epithelial cell targets for in vivo recombinant adenovirus-mediated gene transfer. J. Clin. Invest. 91:225-234.

19. Hazinski, T.A., P.A. Ladd, and C.A. DeMatteo. 1991. Localization and induced expression of fusion genes in the rat lung. Am. J. Respir. Cell Mol. Biol. 4:206-209.

20. Stribling, R., E. Brunette, D. Liggitt, K. Gaensler, and R. Debs. 1992. Aerosol gene delivery in vivo. Proc. Natl. Acad. Sci. USA. 89:11277-11281. 
21. Miller, D.G., M.A. Adam, and A.D. Miller. 1990. Gene transfer by retrovirus vectors occurs only in cells that are actively replicating at the time of infection. Mol. Cell Biol. 10:4239-4242.

22. Janssens, S.P., A. Shimouchi, T. Quertermous, D.B. Bloch, and K.D. Bloch. 1992. Cloning and expression of a cDNA encoding human endotheliumderived relaxing factor/nitric oxide synthase. J. Biol. Chem. 267:14519-14522.

23. Gomez-Foix, A., W. Coats, S. Baque, T. Alam, R. Gerard, and C. Newgard. 1992. Adenovirus-mediated transfer of the muscle glycogen phosphorylase gene into hepatocytes confers altered regulation of glycogen metabolism. $J$. Biol. Chem. 267:25129-25134.

24. Gerard, R., and R. Meidell. 1995. Adenovirus vectors in DNA cloning - a practical approach. In Mammalian Systems. B.D. Hames and D. Glover, editors, Oxford University Press, Oxford. In press.

25. Herz, J., and R.D. Gerard. 1993. Adenovirus-mediated transfer of the low density lipoprotein receptor gene acutely accelerates cholesterol clearance in mice. Proc. Natl. Acad. Sci. USA. 90:2812-2816.

26. Xue, C., A. Rengasamy, T.D.L. Cras, P.A. Koberna, G.C. Dailey, and R.A. Johns. 1994. Distribution of NOS in normoxic vs. hypoxic rat lung: upregulation of NOS by chronic hypoxia. Am. J. Physiol. 267:L667-L678.

27. Janssens, S.P., B.T. Thompson, C.R. Spence and C.A. Hales. 1994. Functional and structural changes with hypoxia in pulmonary circulation of spontaneously hypertensive rats. J. Appl. Physiol. 77:1101-1107.

28. Kawai, N., D.B. Bloch, G. Fillipov, D. Rabkina, H. Suen, P.D. Losty, S.P. Janssens, W.M. Zapol, S. de la Monte, and K.D. Bloch. 1995. Constitutive endothelial nitric oxide synthase gene expression is regulated during lung development. Am. J. Physiol. 268:L589-L595.

29. Archer, S.L., J.P. Tolins, L. Raij, and E.K. Weir. 1989. Hypoxic pulmonary vasoconstriction is enhanced by inhibition of the synthesis of an endothelium derived relaxing factor. Biochem. Biophys. Res. Commun. 164:1198-1205.

30. Crawley, D.E., L. Zhao, M.A. Giembycz, S.F. liu, P.J. Barnes, R.J.D. Winter, and T.W. Evans. 1992. Chronic hypoxia impairs soluble guanylate cyclase-mediated pulmonary arterial relaxation in the rat. Am. J. Physiol. 263: L325-L332.

31. Fineman, J.R., R. Chang, and S.J. Soifer. 1992. EDRF inhibition augments pulmonary hypertension in intact newborn lambs. Am. J. Physiol. 262: H1365-H1371.

32. Dinh-Xuan, A.T., T.W. Higenbottam, C.A. Clelland, J. Pepke-Zaba, G. Cremona, A.Y. Butt, S.R. Large, F.C. Wells, and J. Wallwork. 1991. Impair- ment of endothelium-dependent pulmonary artery relaxation in chronic obstructive lung disease. N. Engl. J. Med. 324:1539-1547.

33. Dinh-Xuan, A.T., J. Pepke-Zaba, A.Y. Butt, G. Cremona, and T.W. Higgenbottam. 1993. Impairment of pulmonary artery endothelium-dependent relaxation in chronic obstructive lung disease is not due to dysfunction of endothelial membrane receptors nor to L-arginine deficiency. Br. J. Pharmacol. 109:587-591.

34. Christman, B.W., C.D. McPherson, and J.H. Newman. 1992. An imbalance between the excretion of thromboxane and prostacyclin metabolites in pulmonary hypertension. N. Engl. J. Med. 327:70-75.

35. Killbourn, R.G., S.S. Gross, J. Adams, A. Jubran, O.W. Griffith, R Levi, and R.F. Lodato. 1990. L-NG-Methylarginine inhibits tumor necrosis factor-induced hypotension: implications for the involvement of nitric oxide. Proc. Natl. Acad. Sci. USA. 87:3629-3632.

36. Sobol, B.J., G. Bottex, C. Emirgil, and H. Gissen. 1963. Gaseous diffusion from alveoli to pulmonary vessels of considerable size. Circ. Res. 13:71-79.

37. Frostell, C., M. Fratacci, J.C. Wain, R. Jones, and W.M. Zapol. 1991. Inhaled nitric oxide: a selective pulmonary vasodilator reversing hypoxic pulmonary vasoconstriction. Circulation. 83:2038-2047.

38. Frostell, C., H. Blomqvist, G. Hedenstierna, J. Lundberg, and W.M. Zapol. 1993. Inhaled nitric oxide selectively reverses human hypoxic pulmonary vasoconstriction without causing systemic vasodilation. Anesthesiology. 78:427435 .

39. Rimar, S., and C.N. Gillis. 1993. Selective pulmonary vasodilatation by inhaled nitric oxide is due to hemoglobin inactivation. Circulation. 88:2884 2887.

40. Pepke-Zaba, J., T.W. Higgenbottam, A.T. Dinh-Xuan, D. Stone, and J. Wallwork. 1991. Inhaled nitric oxide as a cause of selective pulmonary vasodilatation in pulmonary hypertension. Lancet. 338:1173-1174.

41. Girard, C., J. Lehot, J. Pannetier, S. Filley, P. French, and S. Estanove. 1992. Inhaled nitric oxide after mitral valve replacement in patients with chronic pulmonary hypertension. Anesthesiology. 77:880-883.

42. Roberts, J.D., D.M. Polaner, P. Lang, and W.M. Zapol. 1992. Inhaled nitric oxide in persistent pulmonary hypertension of the newborn. Lancet. 340: 818-819.

43. Rossaint, R., K.J. Falke, F. Lopez, K. Slama, U. Pison, and W.M. Zapol. 1993. Inhaled nitric oxide for the adult respiratory distress syndrome. N. Engl. J. Med. 328:399-405. 